\title{
Metabolic syndrome and incidence of asthma in adults: the HUNT study
}

\author{
Ben Michael Brumpton 1,2,3, Carlos Arturo Camargo Jr2,3, \\ Pål Richard Romundstad ${ }^{1}$, Arnulf Langhammer ${ }^{1}$, Yue Chen ${ }^{4}$ and Xiao-Mei Mai ${ }^{1}$
}

Affiliations: 'Dept of Public Health and General Practice, Faculty of Medicine, Norwegian University of Science and Technology, Trondheim, Norway. ${ }^{2}$ Dept of Emergency Medicine, Massachusetts General Hospital, Boston, MA, and ${ }^{3}$ Dept of Epidemiology, Harvard School of Public Health, Boston, MA, USA. ${ }^{4}$ Dept of Epidemiology and Community Medicine, Faculty of Medicine, University of Ottawa, Ottawa, ON, Canada.

Correspondence: B.M. Brumpton, Dept of Public Health and General Practice, Norwegian University of Science and Technology (NTNU), Post box 8905, MTFS, N0-7491 Trondheim, Norway. E-mail: ben.brumptonantnu.no

ABSTRACT Obesity is a risk factor for incident asthma in adults, and obesity is a major component of metabolic syndrome. This study aimed to explore the associations of metabolic syndrome and its components with the cumulative incidence of asthma in adults.

We conducted a prospective cohort study of participants who were asthma-free at baseline $(n=23191)$ in the Nord-Trøndelag Health Study from 1995 to 2008. Baseline metabolic syndrome was categorised using the definition of the Joint Interim Statement from several international organisations. Incident asthma was self-reported at follow-up, which averaged 11 years.

Metabolic syndrome was a risk factor for incident asthma (adjusted OR 1.57, 95\% CI 1.31-1.87). This association was consistent in sensitivity analyses using a stricter asthma definition (adjusted OR 1.42, 95\% CI 1.13-1.79). Among the components of metabolic syndrome, two remained associated with incident asthma after mutual adjustment for the other metabolic components: high waist circumference (adjusted OR 1.62, 95\% CI 1.36-1.94) and elevated glucose or diabetes (adjusted OR 1.43, 95\% CI 1.01-2.04).

Metabolic syndrome and two of its components (high waist circumference and elevated glucose or diabetes) were associated with an increased risk of incident asthma in adults.

@ERSpublications

Metabolic syndrome and two of its components were associated with an increased risk of incident asthma in adults http://ow.ly//p2RNy

This article has supplementary material available from www.erj.ersjournals.com

Received: March 142013 | Accepted after revision: June 182013 | First published online: July 112013

Support statement: This study was supported by the Research Council of Norway (project number 201895/V50) and the Faculty of Medicine, Norwegian University of Science and Technology (NTNU), Trondheim, Norway.

Conflict of interest: Disclosures can be found alongside the online version of this article at www.erj.ersjournals.com 


\section{Introduction}

The prevalence of asthma has been increasing worldwide during the past half century [1]. This increase has been particularly evident in countries such as the UK and the USA $[2,3]$. The increase in asthma prevalence coincided with an increasing prevalence of obesity and, since the late 1990s, researchers have focused their investigations on a possible causal link between these two diseases [4]. Over the past decade, numerous studies have confirmed that obesity is a risk factor for incident asthma in adults [5], with emerging evidence of a distinct asthma phenotype linked to obesity [6, 7].

Obesity is a major component of metabolic syndrome, a cluster of metabolic components that indicate a significantly increased risk of cardiovascular disease [8]. The other components of metabolic syndrome include elevated triglycerides, reduced high-density lipoprotein (HDL) cholesterol, high blood pressure, and elevated glucose or diabetes. To date, few studies have investigated the association of metabolic syndrome or its components with asthma development [9-14], with the notable exception of obesity [5]. Of the limited studies looking at metabolic syndrome or its nonobesity components, one found that metabolic syndrome was associated with asthma-like symptoms [9], while mixed results have been observed for the associations between the other nonobesity metabolic components and asthma [9-14]. Many of these studies, however, cannot assign temporality to the association, due to their cross-sectional design. Research also suggests that the associations between components of metabolic syndrome and asthma may vary by atopic status [11, 15]. We aimed to investigate the prospective associations between metabolic syndrome and its components with incidence of asthma in a large, population-based cohort of adults.

\section{Methods}

Study population

We conducted a prospective study of 23191 adults aged 19-55 years who were free from asthma at baseline. The study population included participants from the county of Nord-Trøndelag, Norway. During 1995-1997, the adult population of Nord-Trøndelag was invited to attend the second survey of the Nord-Trøndelag Health Study (HUNT 2, 1995-1997) [16]. Of the 65237 participants that entered HUNT 2, 37071 also participated in HUNT 3 (2006-2008), to form our study cohort. We then excluded participants with baseline asthma, missing information on asthma, metabolic syndrome or its components at HUNT 2 or HUNT 3, time between last meal and venipuncture, and those aged $>65$ years at follow-up (to reduce misclassification of asthma and chronic obstructive pulmonary disease (COPD)) $[17,18]$. The remainder formed our analysis cohort $(n=23191)$.

\section{Metabolic syndrome}

Waist circumference was measured by a nurse to the nearest centimetre. Blood samples (nonfasting) were taken, and serum separated and refrigerated for analysis. Analysis of triglycerides, HDL cholesterol and glucose were conducted on a Hitachi 911 Autoanalyser with reagents from Boehringer Mannheim (Mannheim, Germany). Blood pressure readings were measured automatically three time using a Dinamap 845XT (Critikon, Tampa, FL, USA), and the mean of the second and third readings were used in the analysis. We defined metabolic syndrome according to the following cut-off points: waist circumference $\geqslant 88 \mathrm{~cm}$ in females and $\geqslant 102 \mathrm{~cm}$ in males; triglycerides $\geqslant 1.7 \mathrm{mmol} \cdot \mathrm{L}^{-1}$; HDL cholesterol $<1.3 \mathrm{mmol} \cdot \mathrm{L}^{-1}$ in females and $<1.0 \mathrm{mmol} \cdot \mathrm{L}^{-1}$ in males; systolic blood pressure $\geqslant 130 \mathrm{mmHg}$ or diastolic blood pressure $\geqslant 85 \mathrm{mmHg}$ or use of anti-hypertensive medication; and glucose $\geqslant 5.6 \mathrm{mmol} \cdot \mathrm{L}^{-1}$ at $\geqslant 4 \mathrm{~h}$ since last meal (serum measurements were nonfasting) or self-reported diabetes. The presence of three or more of these components defined metabolic syndrome. We adopted our definition from the Joint Interim Statement [8], as the definition does not restrict the underlying cause of metabolic syndrome to obesity or insulin resistance, unlike some previous definitions. The following exceptions were included in our definition due to data limitations: we did not have information on cholesterol or glucose-lowering medication use in the main questionnaire; and serum measurements for HUNT 2 were nonfasting, therefore we only used glucose measurements that were taken $\geqslant 4 \mathrm{~h}$ since the last meal.

\section{Covariables}

We collected information on covariates from clinical examinations and questionnaires in HUNT 2, age (19-29, 30-39, 40-49 and 50-55 years), sex (female/male), family history of asthma (yes/no), current smoking status (yes/no), duration of light physical activity $(<1,1-2$ or $\geqslant 3 \mathrm{~h}$ per week), years of education $(<10,10-12$ or $\geqslant 13$ years), receiving social benefits (yes/no), economic difficulties (yes/no), allergic rhinitis (yes/no) and heartburn/acid regurgitation (yes/no). Family history of asthma was defined as any family member (father, mother, brother or sister) with asthma. Social benefit was defined as those that reported receiving sick pay, rehabilitation benefits, retraining benefits, disability pension, old age pension, family income supplement, unemployment benefits, transitional benefits, widow's pension or any other 
benefit. Economic difficulties were defined as those that reported difficulties meeting the cost of food, transport and/or housing. Allergic rhinitis was defined by those who answered yes to the question "Do you have or have you had allergic rhinitis or hay fever?" in the HUNT 3 questionnaire. Heartburn/acid regurgitation was defined by those who reported heartburn/acid regurgitation during the past 12 months at baseline.

\section{Asthma outcome}

The same survey question on asthma was included at baseline and follow-up, "Do you have or have you had asthma?" Cumulative incident asthma was defined from the survey questionnaires as those who did not report asthma at baseline and reported asthma at follow-up (an average of 11 years). Sensitivity analyses included a stricter definition of asthma, i.e. reported asthma and use of asthma medication at follow-up, without report of asthma or wheeze at baseline versus the reference group with no asthma and no wheeze at baseline or follow-up ( $n=20155$ for this analysis). In addition, we further excluded participants who reported COPD at follow-up ( $\mathrm{n}=19980$ for this analysis).

\section{Statistical methods}

Descriptive statistics of included variables were examined in participants with and without metabolic syndrome. We estimated odds ratios and 95\% confidence intervals from multivariable logistic regression models for metabolic syndrome and its components in association with cumulative incident asthma. The odds ratio estimates were adjusted for confounders age, sex and family history of asthma (model 1), and were then additionally adjusted for the covariates (potential confounders) smoking, physical activity, education, social benefit and economic difficulties (model 2), and were then additionally mutually adjusted for all other metabolic components (model 3). Subgroup analyses were conducted by allergic rhinitis (yes, $\mathrm{n}=4367$; no, $\mathrm{n}=13921$; and missing, $\mathrm{n}=4903$ ) as a proxy measure for allergy status, and by heartburn/acid regurgitation (yes, $\mathrm{n}=6321$; no, $\mathrm{n}=15977$; and missing, $\mathrm{n}=893$ ) as a proxy measure for gastro-oesophageal reflux disease (GERD). Finally, we conducted sensitivity analyses using the stricter definition of asthma as described above and further excluded reported COPD cases. The associations between metabolic syndrome and asthma were similar in females and males (see online supplementary materials for sex-specific analyses) and, therefore, we combined sexes in the presentation of results.

\section{Ethics}

The project was approved by the Regional Committees for Medical and Health Research Ethics (Trondheim, Norway). All participants signed informed consent for participation and the use of data in research.

\section{Results}

Characteristics of the participants with $(n=2971)$ and without $(n=20220)$ metabolic syndrome are shown in table 1. Participants with metabolic syndrome were generally older, less physically active, were less educated and were more likely to receive social benefits and to report economic difficulties (sex-specific characteristics of the cohort can be found in online supplementary table S1).

The association between metabolic syndrome and incident asthma is shown in table 2. Metabolic syndrome was associated with incident asthma (OR 1.69, 95\% CI 1.42-2.01 in model 1). After adjustment for all covariates (model 2), the odds ratio was not substantially attenuated (OR 1.57, 95\% CI 1.31-1.87). In sexspecific analyses, the odds ratios for females and males were 1.69 (95\% CI 1.35-2.13) and 1.41 (95\% CI 1.07 to 1.87 ) in model 2, respectively (online supplementary tables S2 and S3). In our subgroup analyses by allergic rhinitis, we observed similar associations between metabolic syndrome and cumulative incidence of asthma in participants with allergic rhinitis (OR 1.44, 95\% CI 1.08-1.94) and participants without allergic rhinitis (OR 1.67, 95\% CI 1.27-2.19) in model 2 (online supplementary table S4). In subgroup analyses stratified by heartburn/acid regurgitation (as a proxy for GERD), we also observed similar associations in subgroups with or without heartburn/acid regurgitation (data not shown). The sensitivity analysis with a stricter definition of incident asthma showed a moderate association consistent with the above findings: OR 1.42 (95\% CI 1.13-1.79) in model 2 (table 3). Further exclusion of those reporting COPD at follow-up did not substantially change our findings (data not shown).

The associations of the components of metabolic syndrome with incident asthma are shown in table 2. Most components showed a weak-to-moderate association with incident asthma in model 1 . However, after adjustment for all covariates and the other metabolic components (model 3), most associations were substantially attenuated. Only the associations for high waist circumference (OR 1.62, 95\% 1.36-1.94) and elevated glucose or diabetes (OR 1.43, 95\% CI 1.01-2.04) remained relatively stable after mutual adjustment for all covariates and the other metabolic components. Similar associations were observed in the sensitivity 
TABLE 1 Characteristics of the analysis cohort participants, Nord-Trøndelag Health Study, Norway

\begin{tabular}{|c|c|c|}
\hline Baseline characteristics & With metabolic syndrome ${ }^{\#}$ & Without metabolic syndrome \\
\hline \multicolumn{3}{|l|}{ Age years } \\
\hline $30-39$ & $715(24.1)$ & $6227(30.8)$ \\
\hline $40-49$ & $1328(44.7)$ & 7849 (38.8) \\
\hline $50-55$ & $648(21.8)$ & $2770(13.7)$ \\
\hline Male & $1537(51.7)$ & $8894(44.0)$ \\
\hline Family history of asthma & $446(16.4)$ & $2931(15.6)$ \\
\hline Smoking & $931(33.0)$ & $5752(30.0)$ \\
\hline \multicolumn{3}{|l|}{ Physical activity h per week } \\
\hline$<1$ & $857(31.6)$ & 4395 (24.3) \\
\hline $1-2$ & $1124(41.4)$ & 7235 (40.0) \\
\hline$\geqslant 13$ & $542(18.4)$ & $5505(27.5)$ \\
\hline Social benefit recipient & $626(26.3)$ & $3337(20.4)$ \\
\hline Economic difficulties & 909 (36.4) & $5465(31.6)$ \\
\hline Allergic rhinitis & $572(24.1)$ & 3795 (23.9) \\
\hline Gastro-oesophageal reflux disease & $1206(52.6)$ & $6324(40.5)$ \\
\hline $\begin{array}{l}\text { Waist circumference } \geqslant 88 \mathrm{~cm} \text { in females, } \geqslant 102 \mathrm{~cm} \\
\text { in males }\end{array}$ & 1779 (59.9) & $1384(6.8)$ \\
\hline Triglycerides $\geqslant 1.7 \mathrm{mmol} \cdot \mathrm{L}^{-1}$ & 324 (91.9) & $4843(24.0)$ \\
\hline $\begin{array}{l}\mathrm{HDL} \text { cholesterol }<1.3 \mathrm{mmol} \cdot \mathrm{L}^{-1} \text { in females, } \\
<1.0 \mathrm{mmol} \cdot \mathrm{L}^{-1} \text { in males }\end{array}$ & $2213(74.5)$ & $2715(13.4)$ \\
\hline $\begin{array}{l}\text { Elevated blood pressure }{ }^{+} \text {or use of } \\
\text { anti-hypertensive medication }\end{array}$ & $2644(89.0)$ & 8765 (43.3) \\
\hline Elevated glucose $\mathrm{s}^{\S}$ or diabetes & $271(9.1)$ & $328(1.6)$ \\
\hline
\end{tabular}

Total $n=23$ 191. Data are presented as $n$ or $n(\%)$. HDL: high-density lipoprotein. ${ }^{\#}$ : metabolic syndrome was defined according to the Joint Interim Statement clinical criteria including alternate indicators anti-hypertensive medication and diabetes; glucose was nonfasting and $\geqslant 4 \mathrm{~h}$ since last meal. ": number of observations does not include total cohort due to missing data. ${ }^{+}$: systolic blood pressure $\geqslant 130 \mathrm{mmHg}$ or diastolic blood pressure $\geqslant 85 \mathrm{mmHg} .{ }^{\S}: \geqslant 5.6 \mathrm{mmol} \cdot \mathrm{L}^{-1}$ and $\geqslant 4 \mathrm{~h}$ since last meal.

analysis using the stricter asthma definition (table 3). The odds ratio for high waist circumference was 1.55 (95\% CI 1.23-1.95) and for elevated glucose or diabetes the odds ratio was 1.64 (95\% CI 1.07-2.52) in model 3. Further excluding of those reporting COPD at follow-up did not change our results (data not shown). By contrast, the other components of metabolic syndrome were not significantly associated with incident asthma.

\section{Discussion}

In this large population-based study, we found that metabolic syndrome was associated with incident asthma in adults. This association was consistent in females and males, and for participants with or without allergic rhinitis. Our study also suggested that high waist circumference and elevated glucose or diabetes were the metabolic components driving this association.

The associations we observed between metabolic syndrome and asthma were consistent with one previous study, which found a moderate association between metabolic syndrome and asthma-like symptoms [9]. In this cross-sectional study of 10038 Korean adults aged 40 to 69 years, participants with metabolic syndrome were more likely to report symptoms of wheeze, resting dyspnoea and post-exercise dyspnoea than their counterparts without metabolic syndrome. Our findings expand on this earlier work by investigating the association prospectively and in subgroups by allergic rhinitis (as a proxy measure for allergy status). Several distinct forms of asthma are recognised (allergic and nonallergic) and the underlying mechanisms can vary between these forms [15]. For example, mechanisms of allergic asthma include adaptive immunity pathways 
TABLE 2 Association (odds ratios) of metabolic syndrome and its components with incident asthma

\begin{tabular}{|c|c|c|c|c|c|}
\hline Metabolic components & Total $\mathbf{n}$ & $\begin{array}{l}\text { Incident } \\
\text { asthma n }\end{array}$ & Model $1{ }^{\#}$ OR $(95 \% \mathrm{Cl})$ & Model $2^{\text {\% }}$ OR $(95 \% \mathrm{Cl})$ & Model $3^{+}$OR $(95 \% \mathrm{Cl})$ \\
\hline $\begin{array}{l}\text { Waist circumference } \\
\geqslant 88 \mathrm{~cm} \text { in females, } \\
\geqslant 102 \mathrm{~cm} \text { in males }\end{array}$ & 3163 & 205 & $1.85(1.57-2.18)$ & $1.73(1.46-2.04)$ & $1.62(1.36-1.94)$ \\
\hline $\begin{array}{l}\text { Triglycerides } \\
\quad \geqslant 1.7 \mathrm{mmol} \cdot \mathrm{L}^{-1}\end{array}$ & 7573 & 324 & $1.31(1.13-1.52)$ & $1.22(1.06-1.42)$ & $1.05(0.90-1.24)$ \\
\hline $\begin{array}{l}\text { Elevated blood } \\
\text { pressure } \text { or use of }^{\S} \text { anti-hypertensive } \\
\text { medication }\end{array}$ & 11409 & 440 & $1.12(0.97-1.29)$ & $1.12(0.97-1.29)$ & $1.03(0.89-1.20)$ \\
\hline
\end{tabular}

Total $n=23$ 191. HDL: high-density lipoprotein; NA: not applicable. " : adjusted for age, sex and family history of asthma. ": adjusted for age, sex, family history of asthma, smoking, physical activity, education, social benefit and economic difficulties at baseline. ${ }^{+}$: adjusted for all covariates and other metabolic risk factors. ${ }^{\S}$ : systolic blood pressure $\geqslant 130 \mathrm{mmHg}$ or diastolic blood pressure $\geqslant 85 \mathrm{mmHg}$. ${ }^{f}$ : elevated glucose was $\geqslant 5.6 \mathrm{mmol} \cdot \mathrm{L}^{-1}$ and $\geqslant 4 \mathrm{~h}$ since last meal. ${ }^{\# \#}$ : metabolic syndrome was defined according to the Joint Interim Statement clinical criteria including alternate indicators anti-hypertensive medication and diabetes; glucose was nonfasting and $\geqslant 4 \mathrm{~h}$ since last meal.

while mechanisms of nonallergic asthma include innate pathways. These forms can also coexist and interact [15]. Our observations suggest that the association between metabolic syndrome and incident asthma in adults does not differ by allergy status.

We found that after mutual adjustment for other metabolic components, the associations between high waist circumference or elevated glucose or diabetes and incident asthma remained. The association between high waist circumference and asthma is consistent with prior studies [19,20], and it is generally accepted that obesity is a risk factor for adult asthma $[5,21]$. The associations of both abdominal and general obesity with incident asthma have previously been investigated in the HUNT 2 cohort, in which incident asthma was associated with both abdominal and general obesity in females and mostly with by general obesity in males [17]. While this previous study attempts to separate the associations between general obesity and abdominal obesity with incident asthma, the present study uses abdominal obesity as a general marker of adiposity. Therefore, the association between high waist circumference and incident asthma in the present analysis reflects both underlying associations, as we did not adjust for body mass index in the analysis.

The association between elevated glucose or diabetes and incident asthma remained after mutual adjustment for the other metabolic components including waist circumference. This association is described less frequently in the literature. Two cross-sectional studies found no association between insulin resistance or elevated glucose and asthma $[9,11]$. By contrast, one cross-sectional study and one prospective study, both from Denmark, reported an association [10,12]. In the prospective study, THUESEN et al. [10] observed that insulin resistance was associated with incident wheezing and asthma-like symptoms after a 5-year follow-up [10]. The Danish population-based study $(n=3441)$ estimated insulin sensitivity using the homeostasis model assessment of insulin resistance (HOMA-IR). The HOMA-IR closely mirrors the glucose clamp technique and has been reported to be a reliable measure of insulin sensitivity for epidemiological studies [22]. However, the outcome variables were based on self-reported symptoms, which might have caused some misclassification of asthma. The inconsistency between the null findings in the former two cross-sectional studies [9, 11] and the positive findings in the studies from Denmark [10, 12], and our current study may be due to the differences in study designs and populations. For example, the prevalence of abdominal obesity was quite different across all five studies, being $\sim 54 \%$ and $7 \%$ in the former two cross-sectional studies $[9,11]$ compared with $\sim 20 \%$ in the studies from Denmark $[10,12]$ and $13 \%$ in the current analysis.

In our study, we found little evidence that the other metabolic components (i.e. elevated triglycerides, reduced HDL cholesterol, and elevated blood pressure or anti-hypertension medication use) were associated 


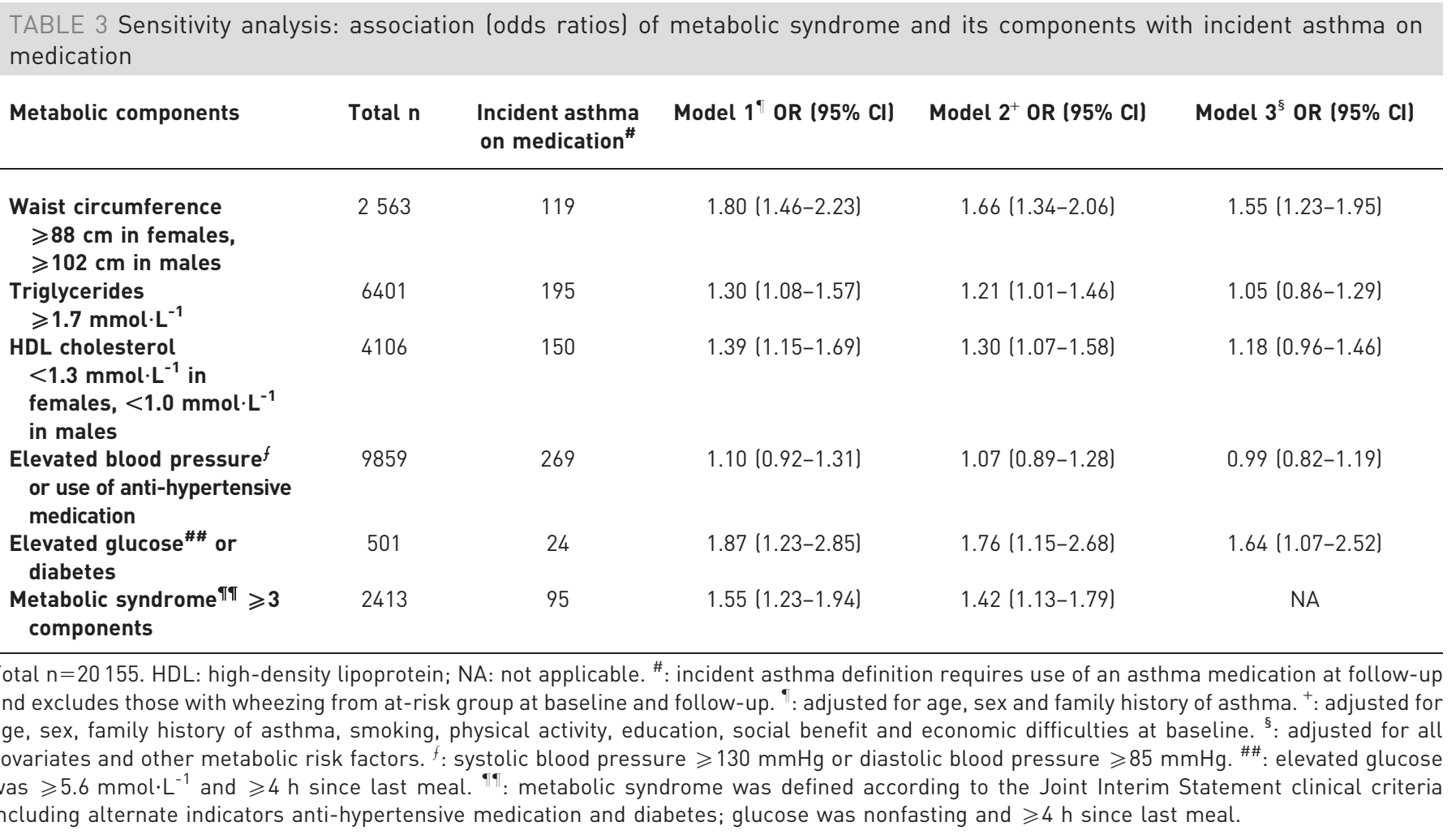

with incident asthma, after mutual adjustment for the other metabolic components. Our results are consistent with some previous studies, including one that did not find an association between elevated triglycerides and asthma [9], and two other studies that found no association between HDL cholesterol and asthma $[9,13]$. However, our study was not consistent with the results from the Korean Health and Genome Study, which found that elevated blood pressure was associated with asthma-like symptoms [9]. This discrepancy may be due to differences in study design (cross-sectional in the Korean Health and Genome Study and prospective in the current study) and study populations, such as the higher mean age in the Korean study (mean age 55 years for subjects with metabolic syndrome) [9], versus the current analysis (mean age 43 years for subjects with metabolic syndrome). Adjusted covariates also differed between the analysis models in the two studies. Further research is needed to clarify the associations between the components of metabolic syndrome and asthma in prospective studies.

There are several possible mechanisms connecting metabolic syndrome and incident asthma. The associations we observed may be driven by obesity, which is thought to change pulmonary physiology, including changes in lung function [23]. Obesity-associated changes in cytokines, chemokines and energy regulating hormones have been suggested to promote local and systemic inflammation and may also play a role [23-25]. The obesity-asthma association may also be due to common genetics shared by both obesity and asthma $[25,26]$. Several potentially confounding comorbidities have also been suggested, including GERD and sleep disordered breathing, which may explain this association $[25,26]$. However, our results remained unchanged in subgroup analysis for participants with and without heartburn/acid regurgitation (as a proxy for GERD). Leaving aside obesity for a moment, the associations between elevated glucose or diabetes and incident asthma is intriguing. It has been hypothesised that systemic low-grade inflammation associated with insulin resistance may be one of the underlying mechanisms of asthma development [10-12], and the association did not seem to be explained by obesity in our study. Although our results support a moderate association between elevated glucose or diabetes and incident asthma, and support earlier findings that linked insulin resistance to asthma and asthma-like symptoms [10, 12], our study was somewhat limited in the investigation of this component.

A weakness of many epidemiological studies of asthma is the definition of asthma. In this study, asthma was defined by a survey questionnaire and further restricted to those who reported asthma, asthma medication use at follow-up and were free from wheeze and asthma at baseline. We were also able to exclude participants who reported COPD at follow-up. Although the diagnosis of asthma has not been confirmed by 
objective means, self-reported asthma has been evaluated as a reliable case finding method for epidemiology studies $[27,28]$. Studies have also observed similar rates of asthma misdiagnosis in obese and nonobese people $[29,30]$. In addition, asthma diagnosis is verified by a physician when asthma medication is prescribed in Norway. Our definition of metabolic syndrome was also limited. The blood serum measures were nonfasting, and both triglycerides and glucose are sensitive to eating, while HDL cholesterol is less affected [31]. To be stringent, we defined elevated glucose levels only when time between last meal and venipuncture was $\geqslant 4 \mathrm{~h}$. Participants with elevated and $<4 \mathrm{~h}$ since their last meal and no reported diabetes were included in the reference group, which could have underestimated the observed association.

We need to be cautious when interpreting the results for the individual components, as the categorisation of these components in the definition of metabolic syndrome are dichotomous and do not allow for accurate adjustment [8]. Further studies should address the individual components in more detail, for example using continuous fasting blood serum levels repeatedly measured over time and incidence of asthma. This would help to remove residual confounding and aid in separating the associations of the individual components of metabolic syndrome with incident asthma. Regarding medication, we only had information on antihypertensive medication use. Medication use may influence two aspects of the results. On the one hand, it may improve the study precision by identifying more cases of metabolic syndrome; on the other hand, adequate medication use may attenuate the associations if the underlying associations are due to elevated serum levels of metabolic components. Despite these study limitations, our study is the largest and longest prospective study to be conducted on the association of metabolic syndrome and its components with incident asthma in adults. The prospective study design allows us to assign temporality.

In this population-based study, metabolic syndrome and two of its components (high waist circumference and elevated glucose or diabetes) were associated with an increased risk of incident asthma in adults. This observation of a link between metabolic syndrome and asthma suggests that clinicians could consider metabolic syndrome as a marker of future risk of asthma. The findings of this study warrant further research, particularly addressing the individual metabolic components and the underlying mechanisms for these associations. Such work could improve the understanding of asthma pathogenesis and thereby assist ongoing efforts to prevent asthma.

\section{Acknowledgements}

The Nord-Trøndelag Health Study (the HUNT Study) is collaboration between the HUNT Research Centre (Faculty of Medicine, Norwegian University of Science and Technology (NTNU), Trondheim, Norway), Nord-Trøndelag County Council and the Norwegian Institute of Public Health.

\section{References}

1 Ekerljung L, Andersson A, Sundblad BM, et al. Has the increase in the prevalence of asthma and respiratory symptoms reached a plateau in Stockholm, Sweden? Int J Tuberc Lung Dis 2010; 14: 764-771.

2 To T, Stanojevic S, Moores G, et al. Global asthma prevalence in adults: findings from the cross-sectional World Health Survey. BMC Public Health 2012; 12: 204.

3 McHugh MK, Symanski E, Pompeii LA, et al. Prevalence of asthma among adult females and males in the United States: Results from the National Health and Nutrition Examination Survey (NHANES), 2001-2004. J Asthma 2009; 46: 759-766.

4 Camargo CA Jr, Weiss ST, Zhang S, et al. Prospective study of body mass index, weight change, and risk of adultonset asthma in women. Arch Intern Med 1999; 159: 2582-2588.

Ford ES. The epidemiology of obesity and asthma. J Allergy Clin Immunol 2005; 115: 897-909.

Lessard A, Turcotte H, Cormier Y, et al. Obesity and asthma: a specific phenotype? Chest 2008; 134: 317-323.

Lugogo NL, Kraft M, Dixion AE. Does obesity produce a distinct asthma phenotype? J Appl Physiol 2010; 108: 729-734. Alberti KG, Eckel RH, Grundy SM, et al. Harmonizing the metabolic syndrome: a joint interim statement of the International Diabetes Federation Task Force on Epidemiology and Prevention; National Heart, Lung, and Blood Institute; American Heart Association; World Heart Federation; International Atherosclerosis Society; and International Association for the Study of Obesity. Circulation 2009; 120: 1640-1645.

9 Lee EJ, In KH, Ha ES, et al. Asthma-like symptoms are increased in the metabolic syndrome. J Asthma 2009; 46: 339-342.

10 Thuesen BH, Husemoen LLN, Hersoug LG, et al. Insulin resistance as a predictor of incident asthma-like symptoms in adults. Clin Exp Allergy 2009; 39: 700-707.

11 Ma J, Xiao L, Knowles SB. Obesity, insulin resistance and the prevalence of atopy and asthma in US adults. Allergy 2010; 65: 1455-1463.

12 Husemoen LL, Glumer C, Lau C, et al. Association of obesity and insulin resistance with asthma and aeroallergen sensitization. Allergy 2008; 63: 575-582.

13 Fessler MB, Massing MW, Spruell B, et al. Novel relationship of serum cholesterol with asthma and wheeze in the United States. J Allergy Clin Immun 2009; 124: 967-974.

14 Al-Shawwa B, Al-Huniti N, Titus G, et al. Hypercholesterolemia is a potential risk factor for asthma. J Asthma 2006; 43: 231-233.

15 Kim HY, DeKruyff RH, Umetsu DT. The many paths to asthma: phenotype shaped by innate and adaptive immunity. Nat Immunol 2010; 11: 577-584. 
16 Krokstad S, Langhammer A, Hveem K, et al. Cohort profile: the HUNT Study, Norway. Int J Epidemiol 2013; 42: 968-977.

17 Brumpton B, Langhammer A, Romundstad P, et al. General and abdominal obesity and incident asthma in adults: the HUNT study. Eur Respir J 2013; 41: 323-329.

18 Mai XM, Langhammer A, Chen Y, et al. Cod liver oil intake and incidence of asthma in Norwegian adults - the HUNT study. Thorax 2013; 68: 25-30.

19 Leone N, Courbon D, Berr C, et al. Abdominal obesity and late-onset asthma: cross-sectional and longitudinal results: the 3C study. Obesity (Silver Spring) 2012; 20: 628-635.

20 Von Behren J, Lipsett M, Horn-Ross PL, et al. Obesity, waist size and prevalence of current asthma in the California Teachers Study cohort. Thorax 2009; 64: 889-893.

21 Beuther DA, Sutherland ER. Overweight, obesity, and incident asthma - a meta-analysis of prospective epidemiologic studies. Am J Respir Crit Care 2007; 175: 661-666.

22 Bonora E, Kiechl S, Willeit J, et al. Insulin resistance as estimated by homeostasis model assessment predicts incident symptomatic cardiovascular disease in Caucasian subjects from the general population: the Bruneck study. Diabetes Care 2007; 30: 318-324.

23 Beuther DA, Weiss ST, Sutherland ER. Obesity and asthma. Am J Respir Crit Care Med 2006; 174: 112-119.

24 Shore SA. Obesity and asthma: possible mechanisms. J Allergy Clin Immunol 2008; 121: 1087-1093.

25 Shore SA, Johnston RA. Obesity and asthma. Pharmacol Ther 2006; 110: 83-102.

26 Beuther DA. Obesity and asthma. Clin Chest Med 2009; 30: 479-488.

27 de Marco R, Cerveri I, Bugiani M, et al. An undetected burden of asthma in Italy: the relationship between clinical and epidemiological diagnosis of asthma. Eur Respir J 1998; 11: 997-997.

28 Toren K, Brisman J, Jarvholm B. Asthma and asthma-like symptoms in adults assessed by questionnaires. A literature review. Chest 1993; 104: 600-608.

29 Pakhale S, Doucette S, Vandemheen K, et al. A comparison of obese and nonobese people with asthma: exploring an asthma-obesity interaction. Chest 2010; 137: 1316-1323.

30 Aaron SD, Vandemheen KL, Boulet LP, et al. Overdiagnosis of asthma in obese and nonobese adults. CMAJ 2008; 179: 1121-1131.

31 Craig SR, Amin RV, Russell DW, et al. Blood cholesterol screening influence of fasting state on cholesterol results and management decisions. J Gen Intern Med 2000; 15: 395-399. 